\title{
An Atypical case of Scleroderma
}

Stavrou C, Wincup CJ, Kravvas G, Manson J

\section{Abstract}

Systemic sclerosis, or scleroderma is a rare systemic autoimmune rheumatic disease with an annual incidence of 3.7 per million in the UK. We report the case of a 55 year old female presenting with insidious onset weight loss, pruritus and worsening shortness of breath. Examination revealed signs of cardiac failure, fine inspiratory crepitations, hepatomegaly and diffuse cutaneous depigmentation although with minimal peripheral sclerodactyly. The anti-nuclear antibodies were interestingly negative and malignancy was not found on thorough screening. The patient was commenced on treatment with oral prednisone and intravenous cyclophosphamide for diffuse cutaneous systemic sclerosis. A rapid response in cardiac function, respiratory symptoms and fatigue was noted. In addition, skin texture rapidly improved with associated re-pigmentation seen within two weeks of commencing treatment. However, within two months of discharge the patient died. The cause of death was thought to be due to cardiac arrhythmia likely secondary to chronic myocarditis.

\section{Case report}

A 55 year old Afro-Caribbean female presented to hospital with recent weight loss, generalised pruritus, malaise and shortness of breath on minimal exertion progressing over recent months. Six months prior to admission she had attended hospital with chest pain and acute shortness of breath. Cardiac enzymes were elevated however the patient declined coronary angiography. Echocardiogram at that time revealed a left ventricular ejection fraction (LVEF) of $55-60 \%$.

Additional past medical history included hypertension and gastro-oesophageal reflux for which she was treated with Ramipril and Ranitidine respectively. In the previous two years she had been reviewed with regards to progressive skin depigmentation and was given a diagnosis of vitiligo.

On examination, she was found to have diffuse areas of depigmentation with perifollicular sparing affecting the arms and torso. Facial and truncal skin tightening was noted in addition to minimal sclerodactyly distally with relative sparing of the finger tips (Figure 1). Cardiovascular examination revealed a mitral pan-systolic murmur with associated palpable right ventricular heave. The jugular venous pressure was elevated and extensive lower limb peripheral oedema was noted. Auscultation of the chest revealed fine bibasal inspiration crepitations. Abdominal examination demonstrated nontender hepatomegaly.

Initial investigations showed raised inflammatory markers; C-reactive protein ( $33 \mathrm{mg} / \mathrm{L} ; \mathrm{NR}<0.6)$ and erythrocyte sedimentation rate $(121 \mathrm{~mm} / \mathrm{hr}$; NR 1-20). Troponin T levels were significantly elevated at $314 \mathrm{ng} / \mathrm{L}$ (NR 0-14). Autoimmune serology interestingly revealed a negative Anti-Nuclear antibody (ANA) although anti-Ro antigens were present. All other auto-antibodies were negative.

Echocardiogram now demonstrated severe mitral regurgitation and impaired systolic function with a LVEF of $<20 \%$. Subsequent investigation with cardiac magnetic resonance imaging (MRI) showed chronic cardiomyopathy felt to be in keeping with advanced scleroderma heart disease. The patient was placed on continuous cardiac monitoring, which noted a short run of non-sustained ventricular tachycardia.

In the context of a negative ANA, malignancy was excluded following extensive investigation including serum tumour markers, Positron Emission Tomography Computerised Tomography (PET$\mathrm{CT}$ ) and bone marrow aspirate and trephine. Skin biopsy demonstrated thickened dermis composed of broad sclerotic collagen bundles and mucin deposition with perivascular inflammation felt to be in keeping with systemic sclerosis.

A diagnosis of atypical diffuse cutaneous systemic sclerosis with associated myocarditis was made. The patient was commenced on treatment with intravenous cyclophosphamide. Low dose oral prednisolone $(10 \mathrm{mg} /$ day $)$, although controversial in the treatment of systemic sclerosis, was given 
in view of poor cardiac function secondary to myocarditis. In addition, bisoprolol, clopidogrel, ramipril, furosemide and spironolactone were started in view of worsening cardiac and renal function.

Following the first dose of cyclophosphamide and low dose oral prednisolone, the patient clinically improved with resolution of the symptoms of cardiac failure. Cardiac enzymes progressively normalised. A rapid improvement was seen in the patient's skin with marked improvements in the degree of thickening, restriction and pigmentation. Unfortunately two months following discharge the patient died unexpected. The case of death was felt to be likely due to an underlying arrhythmia.

\section{Discussion}

Systemic sclerosis (SSc), also known as scleroderma, is a rare systemic autoimmune disease with an annual incidence of 3.7 per million in the UK (Silman A et al, 1988). It carries significant risk of morbidity, although mortality rates have improved over recent years (Nihtyanova SI et al, 2010).

Skin changes are the most noticeable early feature but not all cases present with insidious onset skin thickening at the extremities. The skin can also become hypo/hyperpigmented with a pathognomonic 'salt and pepper' appearance, which can commonly be mistaken for vitiligo (Rai VM \& Balachandran C, 2005). The disease is broadly divided into limited cutaneous systemic sclerosis (ICSSC), in which involvement is predominantly limited to the skin of the hands, forearms and face. Diffuse cutaneous systemic sclerosis (dcSSc) typically affects larger areas of skin and is associated with significant organ involvement (Van den Hoogen $\mathrm{F}$ et al, 2013).

The disease is associated with the presence of anti-nuclear antibodies (ANA) in approximately $90 \%$ of patients (Kavanaugh A et al, 2000). Patients with negative auto-antibodies are a small but well recognised entity. Salazar GA et al (2015) noted that while mortality rates were similar between both groups, vascular complications were more frequently seen in those who were ANA positive whilst lower Gl involvement was more common in ANA negative patients.

Primary myocardial involvement in SSc is a leading cause of mortality and most commonly is asymptomatic; although can present with a wide range of manifestations (Clements PJ et al, 1991). The underlying mechanism for the development of myocarditis is poorly understood but believed to be related to myocardial fibrosis (Kahan A, Allanore Y, 2006). Pieroni M et al (2014) found that early detection of myocardial involvement and prompt initiation of immunosuppressive treatment can prevent long term cardiac damage in most cases.

\section{Learning Points}

- Systemic sclerosis is a rare disease characterised by the presence of anti-nuclear antibodies (ANA). However it is important to consider that the ANA is not positive in all cases and so this should be taken into accounted for when making a diagnosis.

- Screening for common malignancies in patients with atypical systemic sclerosis should be considered (in particular in those who present atypically; for example with a negative ANA).

- Early diagnosis can be difficult as number of conditions can mimic scleroderma (in this case the skin changes were initially attributed to vitiligo). Therefore it is vital to consider any systemic symptoms that the patient may be experiencing.

- Cardiac involvement in systemic sclerosis is common and often asymptomatic. Therefore patients with cardiac symptoms or elevated cardiac enzymes should be investigated thoroughly.

- Early detection and treatment of cardiac involvement can significantly improve long term prognosis.

\section{References}

1. Clements PJ, Lachenbruch PA, Furst DE, Paulus HE, Sterz MG (1991) Cardiac Score. A semiquantitative measure of cardiac involvement that improves prediction of prognosis in systemic sclerosis. Arthritis Rheum 34(11):1371-1380 
2. Kahan A, Allanore $Y$ (2006) Primary myocardial involvement in systemic sclerosis. Rheumatology (Oxford) 45 Suppl 4:iv14-17 (doi: 10.1093/rheumatology/kel312)

3. Kavanaugh A, Tomar R, Reveille J, Solomon DH, Homburger HA (2000) Guidelines for clinical use of the antinuclear antibody test and tests for specific autoantibodies to nuclear antigens. Arch Pathol Lab Med 124(1):71-81

4. Nihtyanova SI, Tang EC, Coghlan JG, Wells AU, Black CM, Denton CP (2010) /mproved survival in systemic sclerosis is associated with better ascertainment of internal organ disease: a retrospective cohort study. QJM 103(2):109-115 (doi: 10.1093/qjmed/hcp174)

5. Pieroni M, De Santis M, Zizzo G et al (2014) Recognizing and treating myocarditis in recentonset systemic sclerosis heart disease: potential utility of immunosuppressive therapy in cardiac damage progression. Semin Arthritis Rheum 43(4):526-535 (doi: 10.1016/j.semarthrit.2013.07.006)

6. Rai VM, Balachandran C (2005) Pseudovitiligo in systemic sclerosis. Dermatol Online J 11(3):41

7. Salazar GA, Assassi S, Wigley $\mathrm{F}$ et al (2015) Antinuclear antibody-negative systemic sclerosis. Semin Arthritis Rheum 44(6):680-686 (doi: 10.1016/j.semarthrit.2014.11.006)

8. Silman A, Jannini S, Symmons D, Bacon P (1988). An epidemiological study of scleroderma in the West Midlands. J Rheumatol 27(4):286-290

9. Van den Hoogen F, Khanna D, Fransen J, Johnson SR, Baron M (2013) 2013 Classification criteria for systemic sclerosis: an American College of Rheumatology/European League against Rheumatism collaborative initiative. Arthritis Rheum 65(11):2737-2747 (doi: 10.1002/art.38098)

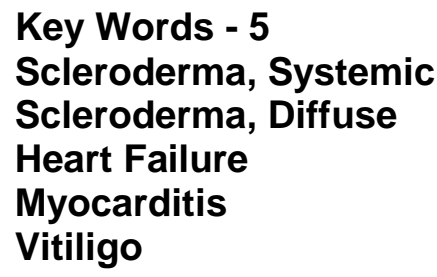

Figure 1- Patient photographs displaying diffuse areas of skin depigmentation with areas of perifollicular pigment sparing affecting the arms and torso, alongside with sclerodactyly.
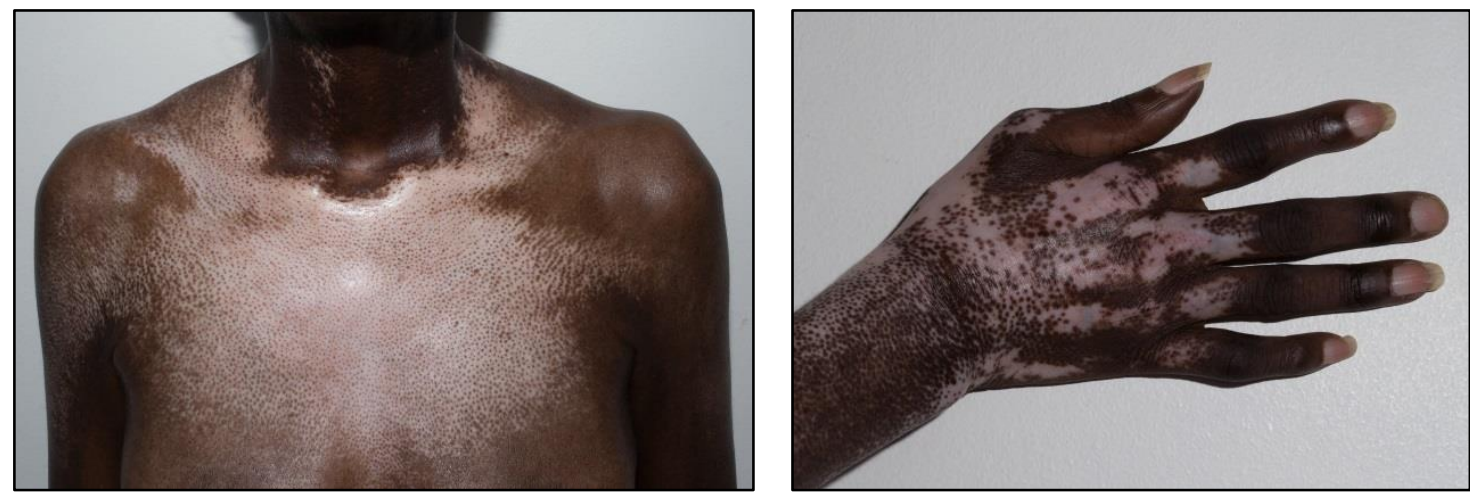